\section{PARAMETRIC MACROMODELING OF INTEGRATED INDUCTORS FOR RF CIRCUIT DESIGN}

\author{
F. Passos, Y. Ye, D. Spina, E. Roca, R. Castro-López, T. \\ Dhaene, F.V. Fernández
}

F. Passos, E. Roca, R. Castro-López and F. V. Fernández are with Universidad de Sevilla, Instituto de microelectrónica de Sevilla, CNM-CSIC, Seville, Spain, e-mail: \{moreira,eli,castro,paco\}@imsecnm.csic.es.

Y. Ye, D. Spina and T. Dhaene are with the Department of Information Technology, Internet Based Communication Networks and Services (IBCN), Ghent University - iMinds, Gent, Belgium, email: \{yinghao.ye, domenico.spina, tom.dhaene\}@intec.ugent.be

Corresponding author: Fabio Passos (moreira@imse-cnm.csic.es)

\begin{abstract}
Nowadays, parametric macromodeling techniques are widely used to describe electromagnetic structures. In this contribution, the application of such parametric macromodeling techniques to the design of integrated inductors and radio-frequency circuit design is investigated. In order to allow such different operations, a new modeling methodology is proposed, which improves the modeling accuracy when compared to former techniques. The new methodology is tailored to the unique characteristics of the devices under study. The obtained parametric macromodel is then used in a synthesis methodology and in the design of a voltage controlled oscillator in a $0.35-\mu m$ CMOS technology.
\end{abstract}

Key words-integrated inductor, parametric macromodels, RF design, single-objective optimization, VCO.

\section{INTRODUCTION}

In recent years many models have been developed for integrated inductors due to their important role in radio frequency integrated circuits (RF ICs). Most of these models are based on lumped-element circuits, like the $\pi$-model [1], the double $\pi$-model [2] and the T-model [3]. However, these models present accuracy issues, with modeling errors that may exceed $10 \%$ [1], [2] or rely on complex extraction techniques from fabricated samples [3].

Nowadays, black-box approaches based on parametric macromodeling techniques establish a reliable solution for studying generic linear and passive electromagnetic (EM) structures [4]-[6]. Parametric macromodeling techniques model the transfer function of such EM structures, which can be expressed in different forms (e.g., admittance, impedance or scattering parameters), preserving at the same time fundamental physical properties, such as causality, stability and passivity, so that a complete characterization both in the frequency and time domains is obtained [5], [6].

The focus of this letter is the modeling of integrated inductors over a large design space, developing a model suitable for different tasks (e.g., inductor synthesis, design space exploration, circuit design, etc.). In this scenario, not only an accurate estimation of the inductors' transfer function is needed (which can later be used to derive the inductor Sparameters and used for circuit design), but also the main inductor performances used in inductor synthesis (inductance $L$ and quality factor $Q$ ) have to be accurately modeled, since these are the performances used by designers to set optimization objectives and constraints.

In this work the parametric macromodeling technique presented in [5] and the sequential sampling strategy described in [6] have been modified and combined to develop a parametric macromodel which is stable and passive over the entire design space. The novelty of this model is given by two different features: first, it allows different levels of accuracy over the design space in order to alleviate the computational cost of building the model in areas where inductors are less suitable for circuit design. Second, the new modeling technique presents an accurate estimation of not only Sparameters but also $L$ and $Q$. The accuracy improvement of all performances is obtained by applying a new error measurement strategy during the sequential sampling technique. This strategy is tailored to the unique characteristics of integrated inductors.

The new modeling methodology which is described in Section 2, is then used in Section 3 to synthetize the inductor required for the design of a voltage controlled oscillator (VCO). To the best of the authors' knowledge, this is the first time a parametric macromodeling technique is used in circuit design. Finally, conclusions are drawn in Section 4.

\section{PARAMETRIC MACROMODELING OF INTEGRATED INDUCTORS}

The integrated spiral inductor topology modeled in this work is shown in Figure 1. The technology selected was a $0.35-\mu \mathrm{m}$ CMOS technology, for which the process information required for EM simulation was available. The methodology presented in this work is completely independent of the inductor topology and technology, being therefore of easy exportability.

Separate models are built for inductors with different number of turns $N$. In each case, the design space for model building is defined by the inner diameter $D_{\text {in }}$ and turn width $w$, parameters that vary in the following ranges: $D_{i n} \in[10,300]$ $\mu \mathrm{m}$ and $w \in[5,25] \mu \mathrm{m}$. The maximum area of the inductors is limited by a reasonably large outer diameter $D_{\text {out }}$ of $400 \mu \mathrm{m}$, whereas the spacing between inductor turns $s$ is maintained fixed at the minimum allowed by the technology, $2.5 \mu \mathrm{m}$, since no performance improvement is achieved by increasing this value [7]. In order to build the models, the S-parameters of the selected samples are evaluated over 17 frequency samples in the range [0.0001, 2.7] GHz. The number of frequency samples used has a direct impact on the accuracy/efficiency trade-off of the modeling process: using more frequency points provides more information about the behavior of the integrated inductor, but it will lead to an increase on computational time, since EM simulations must be performed for each frequency sample. 


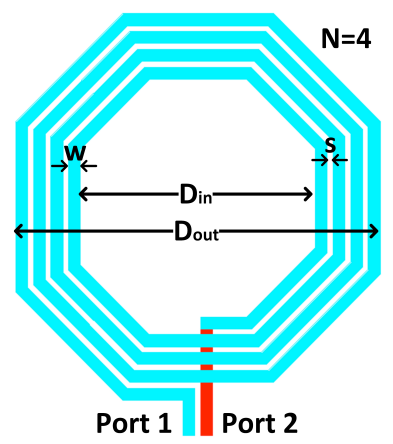

Figure 1 Layout of an octagonal five turns spiral inductor.

The performance parameters $L$ and $Q$ can be directly computed from the inductor S-parameters as:

$$
\begin{gathered}
Z_{e q}=50 \frac{\left(1+S_{11}\right)\left(1+S_{22}\right)-S_{12} S_{21}}{\left(1+S_{11}\right)\left(1+S_{22}\right)+S_{12} S_{21}} \\
L=\frac{\operatorname{Im}\left(Z_{e q}\right)}{2 \pi f} \quad Q=\frac{\operatorname{Im}\left(Z_{e q}\right)}{\operatorname{Re}\left(Z_{e q}\right)}
\end{gathered}
$$

Samples for model construction are evaluated with the EM simulator Keysight's ADS Momentum [8]. Inductances below $50 \mathrm{pH}$ are not considered since such components are not used in $\mathrm{RF}$ design.

The entire modeling technique can be seen in the flowchart presented in Figure 2. The first step to create the parametric macromodel is to electromagnetically simulate the samples corresponding to the corners of the design space and a model is built using these samples. Starting from these initial data samples, a corresponding set of frequency-dependent rational

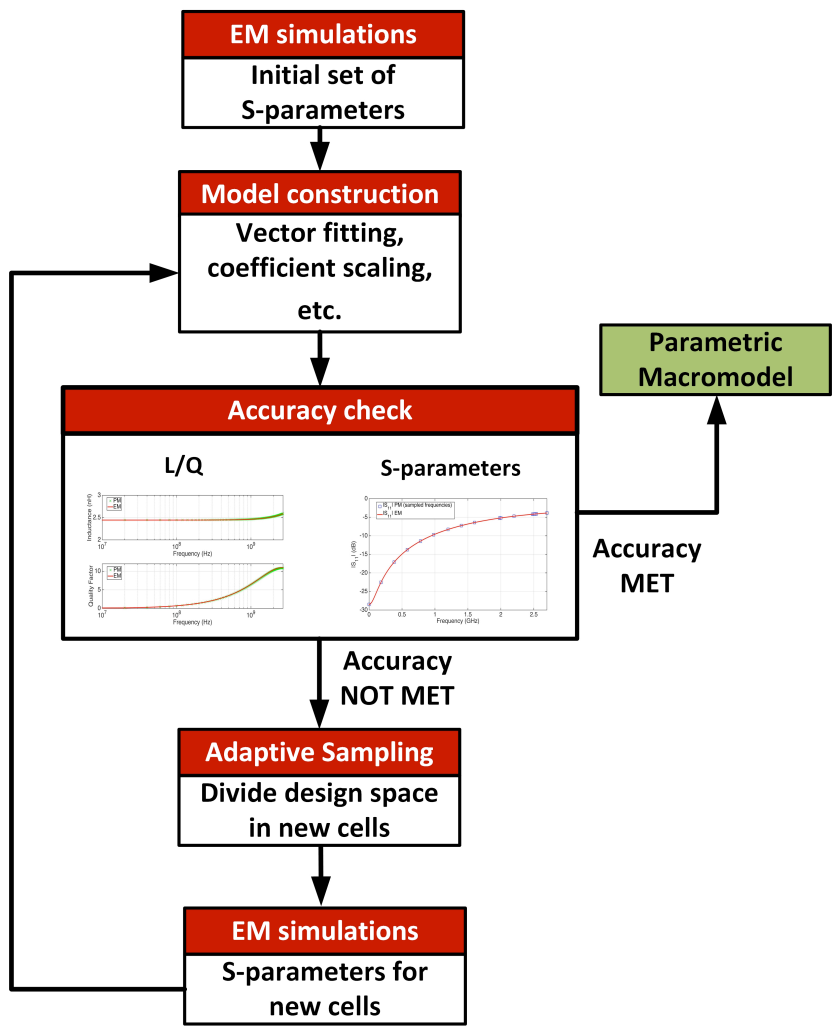

Figure 2 Flowchart for the creation of the proposed parameterized macromodel, with the accuracy check on different parameters.
TABLE 1 Maximum Relative error in the estimation of $L$ and $Q$ at

\begin{tabular}{|c|c|c|c|c|c|}
\hline \multirow{2}{*}{$\mathbf{N}$} & \multirow{2}{*}{$\begin{array}{c}\text { Mabs } \\
\text { (dB) }\end{array}$} & \multicolumn{2}{|c|}{ Model S } & \multicolumn{2}{|c|}{ Model SLQ } \\
\hline & & $M R L(\%)$ & $M R Q(\%)$ & $M R L(\%)$ & $M R Q(\%)$ \\
\hline 1 & -60 & 2.26 & 9.06 & 1.58 & 3.51 \\
\hline 2 & -60 & 2.03 & 5.10 & 2.03 & 5.10 \\
\hline 3 & -50 & 1.45 & 6.31 & 1.45 & 2.85 \\
\hline 4 & -50 & 2.09 & 5.18 & 1.28 & 2.36 \\
\hline 5 & -50 & 1.14 & 2.04 & 1.14 & 2.04 \\
\hline
\end{tabular}
$2.5 \mathrm{GHz}$ for 4936 test inductors.

models is built by means of the vector fitting (VF) technique [9]. After this initial phase, a set of stable and passive frequency-dependent rational models, called root macromodels, is computed. Each of these root macromodels models a single section (cell) of the design space defined by its corners. Afterwards, amplitude and frequency scaling system coefficients are computed for the root macromodels of each cell by means of an optimization procedure and then properly parameterized by positive interpolation operators [5].

Afterwards, the model accuracy of each cell is checked. If the model error is below a defined error threshold for a given cell, the model is marked as accurate enough. If the error is above the selected threshold in a given cell, this cell is divided into subcells by means of an adaptive sampling technique. The whole training-validation process is automatically and recursively applied in each subcell until we have a set of accurate local models that ensure the desired error threshold in every cell of the design space. Merging all the generated local models form the complete parametric macromodel for the entire design space.

It becomes clear that one of the key steps in the modeling process is which error measure to use and its threshold value. In this work, two error measurement techniques will be compared in order to demonstrate the validity of the improved accuracy check proposed in this work. In previous reported models [5], only the maximum absolute error between the Sparameter obtained via EM simulations and the macromodel in the entire frequency range (Mabs) was selected (and for the entire design space). However, the accuracy of $L$ and/or $Q$ can be very sensitive to errors in the estimation of the Sparameters. Table I shows the maximum relative error of $L$ $(M R L)$ and $Q(M R Q)$ at $2.5 \mathrm{GHz}$ calculated over a set of 4936 test inductors (different from the inductors used for model training) when the model is built using only the Mabs error measure of the S-parameters (which we will denote as model S). It should be mentioned that inductors with different number of turns can be modeled with different Mabs thresholds in order to achieve superior accuracy. Note that for inductors up to four turns the $M R Q$ is always higher than $5 \%$. This result confirms that a small error in the estimation of the S-parameters can correspond to a high relative error in e.g., estimating $Q$.

It is therefore clear that a new strategy must be adopted in order to obtain accurate modeling results for $L$ and $Q$. With previous techniques, such as model $\mathrm{S}$, it is impossible to apply different error measurements to different areas of the design space. 
TABLE 2 Number of samples used to build the SLQ model. $L$ and $Q$ mean relative errors for model SLQ (extensive verification).

\begin{tabular}{|c|c|c|c|c|c|c|c|}
\hline \multirow[b]{3}{*}{$\mathbf{N}$} & \multirow{2}{*}{\multicolumn{3}{|c|}{$\begin{array}{c}\text { Number of }\left(W, D_{\text {in }}\right) \text { samples used } \\
\text { for model training and } \\
\text { verification }\end{array}$}} & \multicolumn{4}{|c|}{$\begin{array}{c}\text { Extensive validation } \\
\text { (Offline validation) }\end{array}$} \\
\hline & & & & \multicolumn{2}{|c|}{$100 \mathrm{kHz}$} & \multicolumn{2}{|c|}{$2.5 \mathrm{GHz}$} \\
\hline & Training & $\begin{array}{c}\text { Online } \\
\text { validation }\end{array}$ & $\begin{array}{l}\text { CPU } \\
\text { Time }\end{array}$ & $\begin{array}{l}\overline{\varepsilon_{L}} \\
(\%)\end{array}$ & $\begin{array}{l}\overline{\varepsilon_{Q}} \\
(\%)\end{array}$ & $\begin{array}{l}\overline{\varepsilon_{L}} \\
(\%)\end{array}$ & $\begin{array}{l}\overline{\varepsilon_{Q}} \\
(\%)\end{array}$ \\
\hline 1 & 123 & 88 & $0.94 \mathrm{~h}$ & 0.27 & 1.25 & 0.28 & 0.91 \\
\hline 2 & 253 & 177 & $10.03 \mathrm{~h}$ & 0.20 & 0.74 & 0.21 & 0.66 \\
\hline 3 & 207 & 150 & $11.30 \mathrm{~h}$ & 0.22 & 1.01 & 0.22 & 0.69 \\
\hline 4 & 197 & 145 & $18.81 \mathrm{~h}$ & 0.22 & 0.99 & 0.21 & 0.55 \\
\hline 5 & 140 & 102 & $20.23 \mathrm{~h}$ & 0.22 & 0.88 & 0.19 & 0.46 \\
\hline
\end{tabular}

Thus, the only solution to increase the accuracy of the model is to reduce the error threshold for the Mabs, e.g., from $-50 \mathrm{~dB}$ to $-70 \mathrm{~dB}$. However, this will lead to over-sampling the entire $\left(w, D_{i n}\right)$ design space, which is not a practical solution.

Therefore, the new methodology proposed in this letter consists in adopting an error measure based on S-parameters as well as $L$ and $Q$ and also allow the designer to set different error measures for different areas of the design space. With this approach, we guarantee an accurate estimation not only of the S-parameters, but also of $L$ and $Q$ with the desired accuracy in the entire design space. In this work, the selected relative error threshold values in the estimation of $L$ and $Q$ are:

- $\varepsilon_{L}, \varepsilon_{Q}<6 \%$ for $L \in[0.05,0.5] \mathrm{nH}$;

- $\varepsilon_{L}, \varepsilon_{Q}<3 \%$ for $L>0.5 \mathrm{nH}$.

where $\varepsilon_{\mathrm{L}}$ and $\varepsilon_{\mathrm{Q}}$ are the relative error value of $L$ and $Q$, respectively. The threshold value selected $(3 \%)$ results in a good trade-off between accuracy and computational time (EM simulation of new samples) for the final models. For values of $L<0.5 \mathrm{nH}$, a higher threshold value is considered to reduce the number of EM simulations needed for the model construction, since these inductors are rarely used in circuit design. The parametric macromodels computed by means of the proposed error measure (model SLQ) are capable of modeling the performances of integrated inductors more accurately, therefore helping to improve the design of RF circuits, as will be shown in next section.

The results presented in Table 1 show a significant improvement with respect to the corresponding model $\mathrm{S}$ computed with the same Mabs error threshold for the Sparameters. Furthermore, Table II shows the mean relative error of $L$ and $Q\left(\overline{\varepsilon_{L}}\right.$ and $\left.\overline{\varepsilon_{Q}}\right)$ for an extensive validation study using the 4936 test inductors. The total number of $\left(w, D_{\text {in }}\right)$ samples used to compute the parametric macromodels, including the samples used to verify the model accuracy during the automatic model building stage, are also included in Table 2, as well as the CPU time needed to simulate the inductors used for training and validation.

\section{INDUCTOR SYNTHESIS AND VCO DESIGN}

In this Section a single-differential VCO (illustrated in Figure 3 ) is designed to demonstrate the advantages of the proposed

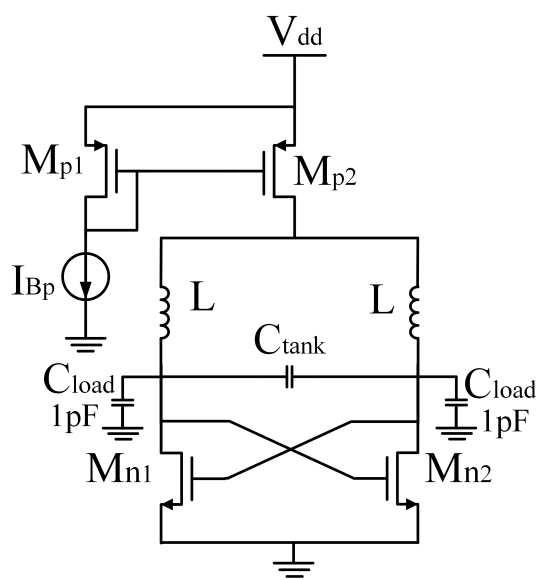

Figure 3 VCO single-differential topology.

modeling strategy (model SLQ) when compared to the former technique (model S) and other methods used by RF designers (e.g., EM simulations).

When designing circuits where inductors are needed (e.g., VCOs), designers usually look for inductors with a given inductance and high quality factors (e.g., by increasing the quality factor of the inductor, the $\mathrm{VCO}$ phase noise is decreased). In order to obtain optimal inductors, an optimization process will be performed using three different performance evaluators: the EM simulator ADS Momentum, the S and the SLQ models. The optimization results and efficiency will then be compared. The optimization algorithm used was PSO [10] (with 40 individuals and 200 generations) and the objective was to achieve an inductor with $L=2 \pm 0.05$ $\mathrm{nH}$ while maximizing the quality factor, within a maximum area of $165 \mu \mathrm{m}^{2}$.

However, in order to properly design inductors for RF circuits, a highly complex set of constraints must be applied.

These constraints are specified in the following set of equations.

$$
\left\{\begin{array}{c}
\text { area }<400 \mu m \times 400 \mu m \\
\left|\frac{L_{@ W F}-L_{@ W F+0.05 G H z}}{L_{@ W F}}\right|<0.01 \\
\left|\frac{L_{@ W F}-L_{@ W F-0.05 G H z}}{L_{@ W F}}\right|<0.01 \\
\left|\frac{L_{@ W F}-L_{\text {at } 0.1 \mathrm{GHz}}}{L_{@ W F}}\right|<0.05 \\
Q_{@ W F+0.05 \mathrm{GHz}}-Q_{@ W F}>0
\end{array}\right.
$$

where, $L_{@ W F}$ and $Q_{@ W F}$ are the inductors' inductance and quality factor at the working frequency $(W F)$ and $L_{@ W F \pm 0.05 \mathrm{GHz}}$ and $Q_{@ W F \pm 0.05 \mathrm{GHz}}$ is the inductance and quality factor at the $W F \pm 0.05 \mathrm{GHz}$. 
TABLE 3 Results for inductor synthesis using three different performance evaluators.

\begin{tabular}{|c|c|c|c|c|c|c|c|c|}
\hline & $\mathbf{N}$ & $\begin{array}{c}\text { Din } \\
(\mu \mathrm{m})\end{array}$ & $\begin{array}{c}w \\
(\mu \mathrm{m})\end{array}$ & $\begin{array}{c}\text { L@2.5 } \\
\text { GHz }\end{array}$ & $\begin{array}{l}\Delta \mathrm{L} \\
(\%)\end{array}$ & $\begin{array}{c}\text { Q@2.5 } \\
\text { GHz }\end{array}$ & $\begin{array}{l}\Delta Q \\
(\%)\end{array}$ & $\begin{array}{l}\text { CPU } \\
\text { time }\end{array}$ \\
\hline EM & 3 & 111 & 7.25 & 1.99 & - & 10.32 & - & $192.2 \mathrm{~h}$ \\
\hline SLQ & 3 & 111 & 7.4 & 1.99 & 0.04 & 10.34 & 0.34 & $4 \mathrm{~min}$ \\
\hline $\mathbf{S}$ & 3 & 110 & 7.6 & 1.98 & 0.71 & 9.75 & 6.21 & $4 \mathrm{~min}$ \\
\hline
\end{tabular}

TABLE 4 Values of the components used in VCO simulation.

\begin{tabular}{cccccccc}
\hline \hline & $\begin{array}{c}\mathbf{W}_{\mathbf{n} 1,2} \\
(\boldsymbol{\mu m})\end{array}$ & $\begin{array}{c}\mathbf{I}_{\mathbf{1} \mathbf{2} 2} \\
(\boldsymbol{\mu m})\end{array}$ & $\begin{array}{c}\mathbf{W}_{\mathbf{p 1}} \\
(\boldsymbol{\mu m})\end{array}$ & $\begin{array}{c}\mathbf{W}_{\mathbf{p} \mathbf{2}} \\
(\boldsymbol{\mu m})\end{array}$ & $\begin{array}{c}\mathbf{I}_{\mathbf{p 1} \mathbf{2}} \\
(\boldsymbol{\mu} \mathbf{m})\end{array}$ & $\begin{array}{c}\mathbf{I}_{\mathbf{B P}} \\
(\mathbf{m A} \mathbf{A})\end{array}$ & $\begin{array}{c}\mathbf{C}_{\text {tank }} \\
(\mathbf{f F})\end{array}$ \\
\cline { 2 - 8 } $\mathbf{E M}$ & 75 & 0.35 & 50 & 150 & 3 & 1 & 397 \\
$\mathbf{S L Q}$ & 75 & 0.35 & 50 & 150 & 3 & 1 & 395 \\
$\mathbf{S}$ & 75 & 0.35 & 50 & 150 & 3 & 1 & 403. \\
\hline \hline
\end{tabular}

TABLE 5 VCO performances using the previously synthetized inductors.

\begin{tabular}{ccccccc}
\hline \hline & $\begin{array}{c}\text { Phase Noise } \\
(\mathbf{d B c} / \mathbf{H z})\end{array}$ & $\begin{array}{c}\Delta \\
(\%)\end{array}$ & $\begin{array}{c}\text { fosc } \\
(\mathbf{G H z})\end{array}$ & $\begin{array}{c}\Delta \\
(\%)\end{array}$ & $\begin{array}{c}\text { Vout } \\
(\mathbf{V})\end{array}$ & $\begin{array}{c}\Delta \\
(\%)\end{array}$ \\
\cline { 2 - 7 } EM & -124.11 & - & 2.50 & - & 1.01 & - \\
SLQ & -124.12 & 0.01 & 2.50 & 0.02 & 1.01 & 0.27 \\
S & -123.68 & 0.37 & 2.50 & 0.36 & 0.97 & 4.40 \\
\hline \hline
\end{tabular}

These constraints are used in order to ensure that the inductance is sufficiently flat from around DC to slightly above the working frequency, that the self-resonance frequency of the inductor is sufficiently above the working frequency and finally, that the deviations in $L$ and $Q$ due to process variations are minimized [7]. These constraints are another reason why such accurate $L$ and $Q$ estimations are needed: with errors of e.g., $6 \%$ (as obtained with the model S), these constraints may be wrongly calculated and optimization may lead to areas where constraints are not actually met.

Table 3 presents the results of the three optimizations using the different performance evaluators. The CPU time needed for each process is also given in Table 3. Notice that the model generation also requires the CPU times shown in Table 2 but that is just a one-time investment and the resulting models can be used as many times as necessary, therefore the time used to build the model should not be accounted for in this optimization.

All techniques achieve similar inductors, however, models $\mathrm{S}$ and SLQ are much more efficient than the optimization using EM simulations. The quality factor of the inductor obtained with model $\mathrm{S}$ is lower than the other methods and the inductance obtained with the model SLQ and the EM simulations is closer to the desired value $(2 \mathrm{nH})$.

Afterwards, the inductors obtained with the models were simulated electromagnetically in order to inspect the model errors (see columns $\Delta L$ and $\Delta Q$ in Table 3). It can be concluded that the optimization using model $\mathrm{S}$ did not converge to the same inductor as model SLQ because of its higher error prediction (around $6 \%$ in $Q$ ). Therefore, using the model $\mathrm{S}$ can lead the RF designer into selecting suboptimal inductors for a given application.

Each of the inductors obtained in the synthesis processes were then used in order to design different VCOs in a $0.35-\mu \mathrm{m}$ CMOS technology with an oscillation frequency (fosc) of 2.5 $\mathrm{GHz}$ and a supply voltage $V_{d d}=1.5 \mathrm{~V}$.

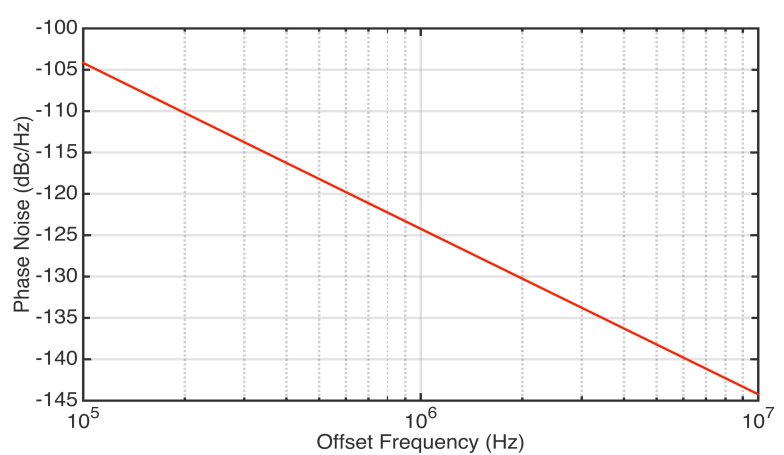

a)

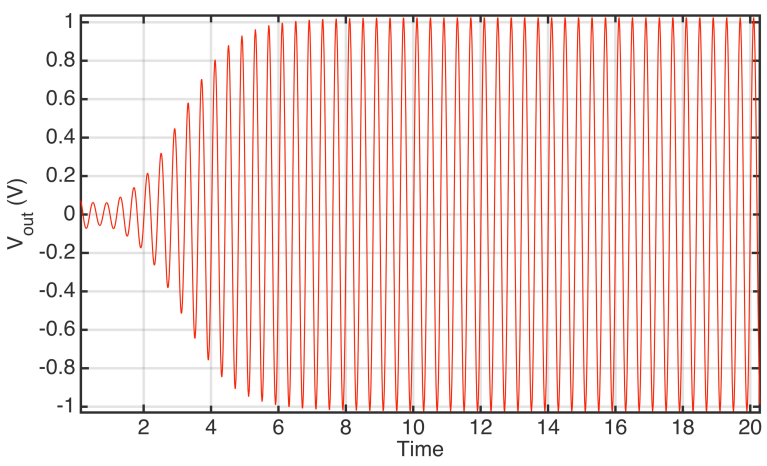

b)

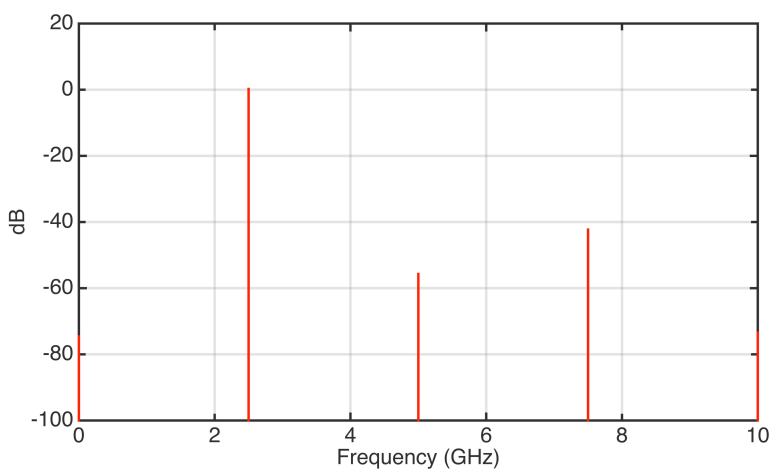

c)

Figure 4 Performances of the VCO designed using the SLQ model. a) VCO phase noise b) VCO transient simulation c) VCO output voltage spectrum.

The design variables of each designed VCOs can be observed in Table 4, where, $W_{n 1,2}$ are the transistor gate widths of the differential pair, $l_{n 1,2}$ are the channel lengths of these transistors, $W_{p 1}, W_{p 2}$ are the gate widths of the current mirror used to polarize the circuit and $l_{p 1}$ and $l_{p 2}$ are their channel lengths. Finally, $C_{l}$ is the capacitor used in the VCO tank. The inductors used in each method are the ones obtained in the synthesis (see Table 3).

Although, the $\mathrm{C}_{\text {tank }}$ value had to be changed among the different VCOs (in order to obtain the desired $f_{\text {osc }}$ ), all the other components were fixed at the same value in order to inspect the influence of the different obtained inductors in the performances of the VCO.

All circuit simulations have been carried out with Cadence SpectreRF circuit simulator [11]. The results are shown in Table 5. It can be observed that the VCO performances are 
superior (lower phase noise and higher output voltage) when the inductor obtained with the model SLQ is used, which is due to the higher quality factor inductor obtained by model SLQ during the optimization stage, which proves that the new modeling strategy brings advantages during circuit design.

The inductors obtained by the models have a certain error, as shown in Table 3. Therefore, the inductors were simulated electromagnetically and also used in circuit simulation in order to compare the VCO performance deviations by using the models. The deviations are shown in Table 5 and denoted by $\Delta$. It can be observed that the model SLQ provides an outstanding accuracy (better than the model S) for all VCO performances, as negligible shifts with respect to the corresponding VCO performances are obtained with a EMsimulated inductor. On the other hand, the VCO performances obtained with the inductor synthesized with model $\mathrm{S}$ show almost $5 \%$ error for the output voltage $\left(\mathrm{V}_{\text {out }}\right)$, which is a key performance (the output voltage of the VCO highly influences the conversion gain of a mixer and its linearity). The performances of the VCO obtained with the model SLQ can be observed in Figure 4, where the phase noise, the transient simulation and the output voltage sepctrum are depicted.

\section{Conclusions}

In this letter a parametric macromodel built with a sequential sampling methodology tailored for the modeling of integrated inductors was presented. This new methodology allows error measures on different performance parameters and increases the time-domain modeling accuracy. An extensive validation study has been performed, proving the improved model accuracy in $L$ and $Q$ estimation (with respect to models based only on S-parameter error measures). The new modeling technique has proven its value by achieving enhanced performances when used in a synthesis methodology and applied to the design of a RF circuit. Furthermore, if automated circuit design methodologies are considered, the model SLQ provides a useful technique for obtaining inductor performances estimation due to its accuracy and efficiency compared to traditional EM simulation.

\section{ACKNOWLEDGMENTS}

This work was supported in part by the TEC2013-45638-C33-R Project (funded by the Spanish Ministry of Economy and Competitiveness and ERDF), and in part by the P12-TIC-1481 Project (funded by Junta de Andalucia).

\section{RefERENCES}

1. C. Yue and S. Wong, "Physical modeling of spiral inductors on silicon," IEEE Transactions on Electron Devices, vol. 47, pp. 560-568, 2000.

2. Y. Cao et al., "Frequency-independent equivalent-circuit model for on-chip spiral inductors," IEEE Journal of Solid-State Circuits, vol.38, no.3, pp. 419-426, 2003.

3. G. Yang, Z. Wang and K. Wang, "Modified T-Model with an improved parameter extraction method for silicon-based spiral inductors," IEEE Transactions on Microwave and Wireless Components Letters, vol.24, no.11, pp. 817-819, 2014.

4. P. Kurgan, S. Koziel and J. W. Bandler, "Low-cost EM-driven surrogate modeling and optimization of planar inductors," IEEE MTT-S International Microwave Symposium, AZ, pp. 1-3, 2015
5. F. Ferranti, L. Knockaert, T. Dhaene, and G. Antonini, "Parametric macromodeling based on amplitude and frequency scaled systems with guaranteed passivity", International Journal Numerical Modelling: El., vol.25, no.2, pp. 139-151, 2012.

6. K. Chemmangat, F. Ferranti, T. Dhaene, L. Knockaert, "Scalable models of microwave system responses using sequential sampling on unstructured grids", International Journal Numerical Modelling: El., vol. 27, pp. 122-137, Jan./Feb, 2014.

7. R. Gonzalez-Echevarria et al., "Automated generation of the optimal performance trade-offs of integrated inductors," IEEE Transactions on Computer-Aided Design of Integrated Circuits and Systems, vol. 33, no. 8, pp. 1269-1273, 2014.

8. ADS Momentum. http://www.keysight.com/en/pc-1887116/momentum3d-planar-em-simulator?cc $=\mathrm{ES} \& \mathrm{lc}=\mathrm{eng}$

9. B. Gustavsen and A. Semlyen, "Rational approximation of frequency domain responses by vector fitting," IEEE Transactions on Power Delivery, vol. 14, no. 3, pp. 1052-1061, July 1999.

10. J. Kennedy and R. Eberhart, "Particle swarm optimization," in Proceedings of IEEE International Conference on Neural Networks, Perth, Australia, vol.4, pp. 1942-1948, 1995.

11. Cadence SpectreRF simulator. https://www.cadence.com/content/cadencewww/global/en_US/home/tools/custom-ic-analog-rf-design/circuitsimulation/spectre-rf-option.html 